\title{
Protein aggregate myopathies: the many faces of an expanding disease group
}

\author{
Rolf Schröder
}

Published online: 6 December 2012

(c) Springer-Verlag Berlin Heidelberg 2012

The physiological functions of proteins are highly dependent on their correct three-dimensional conformation. Disturbances in the proper folding of newly synthesized or pre-existing proteins as well as in pathways responsible for refolding (molecular chaperones) or degradation of misfolded proteins (ubiquitin-proteasome and autophagy systems) may lead to intra- and/or extracellular protein aggregation. These precipitates of misfolded proteins form either ordered (e.g., amyloid fibrils) or disordered (e.g., inclusion bodies) protein aggregates that dissociate only in the presence of high concentrations of detergents or denaturing buffers. Such protein aggregates are the characteristic pathomorphological feature in a wide variety of hereditary and acquired human diseases affecting the central nervous system (e.g., Alzheimer's, Parkinson's disease), the peripheral nervous system (e.g., giant axonal neuropathy, amyloidosis), the eye (e.g., cataracts), the skin (e.g., epidermolytic keratin diseases), the liver (e.g., a1antitrypsin deficiency, alcoholic steatohepatitis), as well as the heart and skeletal muscle (e.g., desmin cardiomyopathy and myopathy).

Protein aggregate myopathies (PAM) are rare familial or sporadic neuromuscular conditions with marked clinical and genetic heterogeneity. Their disease manifestation varies from the neonatal period to late adulthood. They are present in diverse ethnic groups, affect both sexes, and their clinical presentation ranges from congenital myopathies over scapuloperoneal and oculopharyngeal phenotypes to distal, limb girdle, and generalized patterns of muscle weakness and wasting. Non-skeletal muscle

R. Schröder $(\square)$

Institute of Neuropathology, University Hospital Erlangen,

Schwabachanlage 6, 91054 Erlangen, Germany

e-mail: Rolf.schroeder@uk-erlangen.de disease manifestations (e.g., cardiomyopathy, cataracts, skin blistering, frontotemporal dementia), which may precede, coincide with or succeed skeletal muscle weakness, are classically found in distinct forms of hereditary PAM. To date, no causative therapies are available and their progressive clinical course often leads to severe disability and premature death. PAM can be divided into acquired (e.g., inclusion body myositis) and hereditary forms with autosomal-dominant, autosomal-recessive, and X-linked modes of inheritance. In addition, a great number of sporadic and genetically still unresolved cases adds to the genetic complexity. PAM-related gene defects affect the structural and functional role of a wide variety of cellular proteins. The vast majority of PAM are due to mutations in genes coding for cytoskeletal proteins, which are either components of the myofibrillar apparatus $(\alpha-$ actin, myosins, titin, filamin C, myotilin, ZASP, BAG-3, FHL1) or the three-dimensional filamentous extrasarcomeric network (desmin, plectin, $\alpha \mathrm{B}$-crystallin), which interlinks neighboring myofibrils and connects the whole myofibrillar apparatus to myonuclei and the sarcolemma. In addition, mutations in genes coding for proteins with essential functions in protein quality control (VCP, DNAJB6, BAG-3, $\alpha$ B-crystallin), enzymes (GNE), selenoproteins (selenoprotein $\mathrm{N}$ ), and DNA-binding proteins (PABPN1) have been described. This heterogeneous list of PAM-related gene defects will likely contain many more members due to the rapid progress of next generation sequencing and proteomic approaches.

Is the clinical and genetic complexity of PAM also mirrored on the myopathological level? Though this issue is far away from being simple, skeletal muscle tissue seems only to have a limited number of morphological responses to various gene defects. The majority of PAM causing gene defects (desmin, plectin, $\alpha \mathrm{B}$-crystallin, filamin $\mathrm{C}$, myotilin, 
ZASP, BAG-3, FHL1) result in myofibrillar myopathies (MFM), which are basically characterized by the presence of sarcoplasmic desmin-positive protein aggregates and degenerative changes of the myofibrillar apparatus. A second, in-itself heterogeneous group of PAM shows sarcoplasmic ( $\alpha$-actin, myosins, GNE) and/or nuclear $(\alpha$-actin, PABPN1) protein aggregates (e.g., thin filaments, caps, intranuclear rods, zebra bodies, hyaline bodies) with distinct (desmin-negative) patterns of immunoreactivity that clearly differ from MFM lesions. In a third group (DNAJB6, VCP, selenoprotein N, titin), it is still a matter of debate (and limited data) if - and to what extent-these PAM belong to the MFM subgroup. Regarding the myopathological presentation of PAM, it is further noteworthy that the extent of protein aggregation and concomitant myodegeneration can be highly variable even in patients carrying identical mutations (e.g., desmin) and that certain mutations in PAM-related genes (e.g., plectin) may also result in myopathies without protein aggregates or nonmuscle phenotypes. Thus, the complexity of clinical, morphological, and genetic data makes the future establishment of a comprehensive classification of PAM an ambitious goal to achieve.

What are the precise molecular pathways and sequential steps that lead from an individual PAM-related gene defect to pathological protein aggregation and progressive muscle damage? The final answers to this question are still not available and, obviously, the structural, biomechanical, and biochemical basis of progressive muscle damage in distinct PAM cannot be attributed to a simple pathophysiological explanation. Recent years have provided a multitude of exciting novel insights into the molecular pathogenesis of PAM. As a result, complex disease concepts emerge in which PAM-related gene defects cause effects on multiple levels by influencing the structure and function of the sarcomeric and extrasarcomeric cytoskeleton, signaling cascades, protein quality control systems as well as the function of cell organelles. Thus, not a single but a combination of pathogenic events finally leads to a deleterious effect on muscle cells.

The current issue of the Acta Neuropathologica provides five comprehensive reviews on PAM due to mutations in genes coding for $\alpha$-actin, myosins, filamin $\mathrm{C}$, desmin, and plectin. In addition to the many clinical, genetic and myopathological faces of these hereditary myopathies with protein aggregation, the five reviews provide deeper and up to date insights into individual pathophysiological concepts derived from in vitro studies as well as cell and animal models. 